This item was submitted to Loughborough's Research Repository by the author.

Items in Figshare are protected by copyright, with all rights reserved, unless otherwise indicated.

\title{
Exercise and taurine in inflammation, cognition, and peripheral markers of blood-brain barrier integrity in older women
}

PLEASE CITE THE PUBLISHED VERSION

https://doi.org/10.1139/apnm-2017-0775

\section{PUBLISHER}

NRC Research Press (C) The Authors

VERSION

AM (Accepted Manuscript)

\section{PUBLISHER STATEMENT}

This work is made available according to the conditions of the Creative Commons Attribution-NonCommercialNoDerivatives 4.0 International (CC BY-NC-ND 4.0) licence. Full details of this licence are available at: https://creativecommons.org/licenses/by-nc-nd/4.0/

\section{LICENCE}

CC BY-NC-ND 4.0

\section{REPOSITORY RECORD}

Chupel, Matheus Uba, Luciele G. Minuzzi, Guilherme Eustaquio Furtado, Mario L. Santos, Eef Hogervorst, Edith Filaire, and Ana Maria Teixeira. 2019. "Exercise and Taurine in Inflammation, Cognition, and Peripheral Markers of Blood-brain Barrier Integrity in Older Women". figshare. https://hdl.handle.net/2134/33169. 
Exercise and taurine in inflammation, cognition, and peripheral markers of blood-brain barrier integrity in older women

Authors:

Matheus Uba Chupel ${ }^{1}$, matheusuba@hotmail.com

Luciele Guerra Minuzzi ${ }^{1}$

lucielegm@gmail.com

Guilherme Furtado ${ }^{1}$

furts2001@yahoo.com.br

Mário Leonardo Santos ${ }^{1}$

czleo8@gmail.com

Eef Hogervorst ${ }^{2}$

e.hogervorst@1boro.ac.uk

Edith Filaire ${ }^{3}$

edith.filaire@univ-orleans.fr

Ana Maria Teixeira ${ }^{1}$

ateixeira@fcdef.uc.pt

Addresses:

${ }^{1}$ Faculty of Sport Science and Physical Education, University of Coimbra.

Estádio Universitário de Coimbra, Avenida Conímbriga, Pavilhão 3. 3040-248, Coimbra, Portugal

${ }^{2}$ Applied Cognitive Research NCSEM Loughborough University, Loughborough UK.

Epinal Way, Loughborough, LE11 3TU, Leicestershire, UK.

${ }^{3}$ CIAMS, Université d'Orléans, Orléans, France; CIAMS, Université Paris-Sud, Université ParisSaclay, Orsay, France; UMR 1019, INRA, Equipe ECREIN UNH, Clermont-Ferrand, France. Allée 2 du Château, 45067, Orléans, France.

\section{Corresponding author:}

Matheus Uba Chupel

Estádio Universitário de Coimbra, Avenida Conímbriga, Pav 3, 3040-248, Coimbra, Portugal

Phone Number: (351)239 802 770. e-mail: matheusuba@,hotmail.com 


\section{Abstract:}

Immunosenescence contribute to increase the blood-brain barrier (BBB) permeability, leading cognitive impairment and neurodegeneration. Thus, we investigated the anti-inflammatory effect of exercise and taurine supplementation on peripheral markers of blood-brain barrier, inflammation, and cognition of elderly women. Forty-eight elderly women $(83.58 \pm 6.9$ years $)$ participated in the study, and were allocated into combined exercise training (CET: $n=13)$, taurine supplementation (TAU: $n=12$ ), exercise training associated with taurine (CET+TAU: $n=11)$, or control group (CG: $\mathrm{n}=12$ ). Exercise was applied twice a week (multi-modal exercise). Taurine ingestion was $1.5 \mathrm{~g}$., once a day. Participants were evaluated before and after 14-weeks of intervention. Plasma levels of IL-1 $\beta$, IL-1ra, IL-6, IL-10, IL-17, TNF- $\alpha$, and serum concentration of S100 $\beta$ and neuron specific enolase (NSE) were determined. The mini mental state examination (MMSE) was also applied. Concentrations of S100 $\beta$ were maintained in all intervention groups, while a subtle increase in the CG was found. NSE levels increased only in TAU group $(p<0.05)$. CET reduced TNF- $\alpha$, IL-6, and IL-1 $\beta /$ IL-1ra, IL-6/IL10, TNF- $\alpha /$ IL-10 ratios $(p<0.05)$. TAU decreased the IL-1 $\beta /$ IL-1ra ratio $(p<0.05)$. MMSE score increased only in CET+TAU group $(\mathrm{p}<0.05)$. Multiple regression analysis showed that there was a trend for changes in IL-1 $\beta$ and CCI to be independently associated with changes in S100ß. Exercise and taurine decreased inflammation, and maintained the BBB integrity in elderly women. Exercise emerged as an important tool to improve brain health even when started at advanced ages.

Key-words: aging; inflammation; supplementation; taurine; brain; cognition; 


\section{INTRODUCTION}

Chronic inflammation is involved in the pathophysiology of many neurodegenerative conditions (Cho et al. 2015). Related to the genesis and worsening of these is the increased blood-brain barrier (BBB) permeability, an endothelial structure which controls the influx and efflux of components between blood flow and central nervous system (CNS) (Abbott 2000). Low chronic systemic inflammation during aging, known as 'inflammaging', has been proposed to increase BBB permeability (Elwood et al. 2017), which has been measured through peripheral levels of neuronalderived proteins, such as S100ß and neuronal-specific enolase (NSE) (Marchi et al. 2004, Sağ et al. 2017). S100 $\beta$ protein is composed by two isometric subunits being expressed primarily by astrocytes and found predominately in astroglial and Scwann cells, while NSE is a 78kD gammahomodimer that represents the mainly enolase-enzyme found in neurons (Kanner et al. 2003, Haque et al. 2016). Quantitative measures of both biomarkers have been used to represent BBB permeability and neuronal damage in humans, respectively (Isgrò et al. 2015, Dadas et al. 2016). Epidemiological evidence has shown that physical activity can attenuate this age-related immune suppression and ameliorate health status in older participants (Beavers et al. 2010). Combined exercise training (CET) has demonstrated to be effective in increasing physical fitness and cognition (Vaughan et al. 2014). Nutritional therapy, such as supplementation with taurine, can also decrease inflammation in several pathological conditions (Marcinkiewicz and Kontny 2014). While it is one of the most abundant amino acids in the human body, taurine levels decrease with age (Redmond et al. 1998). Animal and human model shave shown that both exercise and supplementation with taurine have beneficial effects in modulating the Th1/Th2 cytokine balance and down-regulate the expression of pro-inflammatory mediators related to endothelial dysfunction (Marcinkiewicz and Kontny 2014, Chupel et al. 2017). However, the combination of exercise training and supplementation with taurine on inflammatory parameters have not yet been investigated in older subjects. The purpose of the present study was to examine the effect of both exercise and taurine, administrated in combination and isolated on peripheral markers of BBB 
dysfunction, inflammatory balance, and cognition in older women. It is expected that exercise and supplementation with taurine will lead to a decrease in peripheral markers of BBB permeability, together with an improvement of an anti-inflammatory environment and increase in cognition.

\section{MATERIALS AND METHODS}

\section{Study Design}

This was a naturalistic, prospective, controlled trial (treatment vs care as usual). All subjects volunteered to participate in exercise classes or supplementation programs. The sample size was estimated using adjusted ANOVA for repeated measures effects using the G*Power software (version 3.1.9.2). Alpha (type I error rate) was set at 0.05 , and power (type II error rate) at 0.85 with the inclusion of forty participants revealing sufficient power.

\section{Participants}

Forty-eight older women $(83.5 \pm 6.9$ year old $)$ participated in the study and were allocated into four groups: a combined exercise training (CET: $n=13)$, supplementation with taurine (TAU: $n=12$ ), a group undergoing CET plus supplementation (CET+TAU: $n=11$ ), and a control group receiving care as usual (CG: $n=12$ ). Details of the experiment design are presented in Figure 1 and the participants characteristics are shown in Table 1. All of them completed and signed an informed consent form prior to their enrolment in the study. After obtaining this consent, data was collected regarding biosocial, global health and general medical history with the medical staff of the nursing home. To be eligible for the study, participants needed to fulfil the following criteria: being a woman living in a nursing home support centre; age $>60$ years; capacity to practice exercise without causing harm to themselves with approval of the medical department. Based on the initial assessment, we excluded volunteers with history of recent head trauma, uncontrolled diabetes mellitus and hypertension, current chronic renal, liver or respiratory disorders, neurologic disorders or those with severe cognitive impairment according to the cut-off Mini Mental State Examination criteria (MMSE) ( $\leq 9$ points), recent myocardial infarction or stroke (within the previous 6 months). We also excluded those individuals who were on hormone replacement therapy. Individuals with 
unstable medical conditions, highlighted by starting new medications within the data collection period were also excluded. The remaining subjects were allowed to continue using their usual prescribed medication (use of furosemide, valsartan, fluoxetine and alprazolam occurred). Comorbidities are present among the sample such as: hypertension (75\%), heart failure (58\%) and dyslipidaemia (34\%). This study was approved by the Faculty of Sport Science and Physical Education- University of Coimbra Ethical Committee, and conducted according to the Declaration of Helsinki guidelines on research in humans.

\section{Experimental Design}

All participants in the study lived in the same institution, and were evaluated before and after 14 weeks of intervention. CET was applied twice a week and the classes took place inside the nursing room gym, on nonconsecutive days, during fourteen weeks. The TAU group was supplemented during the same period. CET+TAU associated both interventions (exercise + supplementation), and the CG did not follow any type of exercise program during 14 weeks but received care as usual.

\section{Experimental Procedures}

\section{Exercise}

The development of the combined exercise training program (CET) was conducted by specialists in exercise prescriptions for the older population. The method designed for the CET program followed the recommendations of the American College of Sport Medicine/American Heart Association for the organization of exercise interventions (Nelson et al. 2007). To be safe for an older population, CET was a chair-based exercise program. The duration of each class was 60 minutes involving six minutes of warm-up, forty-eight minutes consisting of a main part and six minutes of cool-down. CET classes comprised resistance exercise and aerobic/cardiovascular training. Two sessions of pre-intervention familiarization occurred to adapt participants to the resistance training which involved the major muscle groups, and was performed by a predetermined number of exercises that ranged from 4-8 activities, 2-3 sets with15-20 repetitions. The cadence of execution was 2:2 and rest between sets ranged from 30-45 seconds (example of activities: front squat, unilateral hip 
flexion, bench over row, chest press, standing reverse fly, spine twist extension arm, shoulder press/twist arm front position, frontal total raiser, biceps arm curl stand/chair, overhead triceps exertion). The aerobic/cardiovascular component was held in series using 4-8 chair-based exercises and walking around the room (with and without obstacles). Exercises included simulated walking, walking around the gym-room, hip marching, chair-stand and upper body twist, standing rear leg extension, step heel, and kicks in a seated position. The CET program was divided into two microcycles (microcycle1 during the first seven weeks; microcycle2 the remaining weeks). Resistance training progressed within microcycles using decreased rest between sets, increased time of muscle contraction, and use of more complex progressions in exercises, while in the aerobic part walking with obstacles and with changes of direction were introduced. The participants did the resistance training using the yellow color elastic-band (TheraBand, Hygenic Corporation, Akron, $\mathrm{OH}, \mathrm{USA}$ ). The exercise intensity was measured using the OMNI perceived exertion scale (PES), which consisted of a scale ranging from 0 to 10 points concerning the quality of efforts:(0) extremely (1-2) easy, (3-5) somewhat easy, (6-7) somewhat-hard; (8) hard (9-10) extremely-hard). The intensity was kept within values of PES, ranging from 5 to 7 points. In addition, exercise intensity was monitored using heart rate monitors (Polar, 810), which were randomly distributed between the participants during the exercise program to record any abnormal alterations on heart rate and to avoid cardiovascular risk. No music was used during the exercise classes.

\section{Supplementation}

Taurine was supplied by LaborSpirit LTDA, (Portugal). The dosage used was $1.5 \mathrm{~g}$, which is well tolerated and safe for humans, and has been shown effective in decreasing inflammation and improving cardiac function in older people (Shao and Hathcock 2008, Ahmadian et al. 2017). Taurine was diluted in $150 \mathrm{~mL}$ of drinking water once a day during 14 weeks. Supplementation was given every day in the morning, and subjects of both supplement (TAU and CET+TAU) groups received the supplementation at the same time of the day. In the days when there were CET sessions supplementation was ingested at least 30 minutes before the session. In total, each subject received 
98 doses of $1.5 \mathrm{~g}$ of taurine over a period of 14 weeks. Only the principal researcher and the staff of the nursing home had access to the supplement. The caregivers were responsible to dilute the powder in water and give it to the subjects, recording when the supplemented was ingested. The staff was oriented regarding the correct administration of the supplement, clarification of doubts concerning their use, and to stop the procedure in the presence of any adverse side-effect.

\section{Data Collection}

All baseline evaluations were performed during the morning, two weeks before the beginning of the interventions. Evaluations occurred on separated days, beginning with the assessments of the anthropometric measures, the cognitive profile, and the hand-grip test. Collection of blood samples was done on a separate day by a registered nurse. The participants were warned two days in advance for the need to wear appropriate clothes, to avoid ingestion of alcohol and caffeine, and to maintain their sleeping habits. All data collection was done by a staff member and an assistant, and took place individually in a separate and quiet room (with adequate light and temperature) to maintain the participants' privacy. To avoid interference of tester variability in data collection, each participant was always evaluated by the same staff members at the same time of day.

\section{Global Health}

In order to determine the presence of comorbidities in participants, all data regarding global health were obtained by interview from the medical department of the social and health care instituteand by applying the Charlson Comorbidity Index (CCI). Based on 17 comorbid conditions, this index is reported to be able to predict 1 to 10-year mortality risk (Charlson et al. 1994).

\section{Anthropometric Measures}

Body mass was determined using a portable scale (Seca, model 770, Germany) with a precision of 0.1 kilograms. A retractable glass fiber tape (Hoechstmass-Rollfix, Germany) was used to measure the waist circumference and stature was determined using a portable stadiometer (SecaBodymeter, model 208, Germany) with 0.1 centimeters of precision.

\section{Nutritional Assessment}


The mini nutritional assessment (MNA) was used to evaluate the nutritional status, and was fulfilled by the participant together with the nursing staff. It is composed by a total of 18 items and the score ranges from 0 to 30 . The MNA was used to classify the nutritional status of the elderly among a state of protein-energy malnutrition ( $<17$ points), risk of malnutrition (between 17 and 23.5 points), and satisfactory nutritional status ( $>23.5$ points) (Guigoz et al. 1996). Additionally, we consulted the nutritionist menus developed for the nursing home, to verify the amount of macronutrients intake during the intervention.

\section{Cognitive Assessment}

The evaluation of cognitive function was made using the Mini Mental State Examination (MMSE). The MMSE is a 30-point scale instrument that assesses five areas of cognition: orientation, immediate recall, attention and calculation, delayed recall, and language (Folstein et al. 1975). It is used to classify individuals by cognitive profile, as: [0-9] severe cognitive impairment; [10-18] moderate cognitive impairment; [19-24] mild cognitive impairment; [25-30] normal cognitive profile. The validated Portuguese version was used (Morgado et al. 2009).

\section{Hand-Grip Strength Test (HGT)}

Hand-Grip strength was measured using a dynamometer with registration in kilograms (Lafayette, 78010, Indiana, USA). The participant was in a standing position with the elbows positioned at the side of the body. The handle was appropriately regulated to their hand size. When ready, the subject squeezed the dynamometer with maximum isometric effort, which was maintained during five seconds. Thirty seconds of rest between measurements for both hands were given. The test was performed twice. The best value of each hand was computed and the higher value obtained was used.

\section{Blood Collection and Analyses}

Blood samples were collected during the morning (from 10 a.m. to 11 a.m.) by venipuncture, after fifteen minutes of rest, at baseline and after fourteen weeks. All the subjects were encouraged to avoid strenuous physical efforts in the two days prior to the blood collection. Samples were drawn 
into vacutainer tubes containing EDTA for plasma and gel separator for serum analytics. After processing, plasma and serum were aliquoted into eppendorfs and stored at $-80^{\circ} \mathrm{C}$ until determination of the plasma concentrations of IL-1 $\beta$, IL-1ra, IL-6, TNF- $\alpha$, IL-10 (Invitrogen, CA) and IL-17 (R\&D Systems, UK) by ELISA. Serum samples were used to determination of peripheral markers of BBB permeability, S100 $\beta$ and neuronal specific enolase (NSE) concentrations, by ELISA (Biovendor Laboratory Medicine, Czech Republic and R\&D Systems, UK, respectively). Samples were run in duplicate and diluted when appropriate according to the manufacturer instructions. The intra-assay coefficient of variability was $2.7 \%$ for IL-1 $\beta, 3.2 \%$ for IL-1ra, $4.9 \%$ for TNF- $\alpha, 5.3 \%$ for IL-10, $2.3 \%$ for IL-17, $7.3 \%$ for S100 $\beta$ and $2.4 \%$ for NSE. The inter-assay CV was less than $10 \%$ for all biological analysis.

\section{Statistical Analyses}

Descriptive statistics were presented as mean \pm standard deviation for all variables. Data were logconverted to reduce bias arising from non-uniformity error. Analysis of variance using repeated measures ANOVA were performed to examine the response to the interventions. The within measures effect was time (before-after intervention) and between measures effect was treatment (4 types). Bonferroni test was applied post hoc to detect differences between groups. The percent of change after the interventions was calculated ([postvalue/prevalue]-1) and a multiple regression analysis using interleukins changes $(\Delta \mathrm{IL}-1 \beta, \Delta \mathrm{IL}-1 \mathrm{ra}, \Delta \mathrm{IL}-6, \Delta \mathrm{IL}-10, \Delta \mathrm{IL}-17$ and $\Delta \mathrm{TNF}-\alpha)$, and baseline CCI was performed to explore their influence on changes in S100 $\beta$ over time. Those variables that did not contribute to the adjusted model were removed by stepwise backward analysis. The coefficient for each variable was obtained from the adjusted model with the lowest $p$ value. Comparison between groups at baseline were performed using one-way ANOVA. Additionally, univariate analysis for within groups comparisons in were done using the student ttests for paired samples. To report the strength of the exercise interventions, the effect size (pointbiserial correlation, $r_{\mathrm{pb}}$ ) was calculated and converted to standardized Cohen's $d$ changes in means, categorized as trivial $(d<0.2)$, small $(d \geq 0.2$ to $\leq 0.6)$, moderate $(d \geq 0.6$ to $\leq 1.2)$, large $(d \geq 1.2$ to $\leq$ 
$2.0)$, very large $(d \geq 2.0$ to $\leq 4.0)$ and nearly perfect $(d>4.0)$ (Hopkins et al. 2009). All statistical analysis were performed using SPSS (IBM, Statistics, version 23) and level of significance was set at $p<0.05$.

\section{RESULTS}

No adverse events, injury or complications related to the interventions were seen during the study. From those 76 women who started the interventions, 28 were not re-evaluated at follow-up (10 of them discontinued the intervention, and 18 were excluded of the analysis due less than $70 \%$ of minimum adherence established). People who dropped out were more likely to be less educated, presented with more co-morbidity, and had a lower cognitive score than those who finished the trial (from 23 participants who dropped out: only $34 \%$ had completed the primary school, and around $30.3 \%$ had more than five comorbidities, and mean levels of MMSE was 15.6). Details of sample drop-out are provided in figure 1. No differences regarding age, anthropometric variables and cognitive profile were found among the four groups who completed the trials. According to the nursing home menu implemented during the entire study, the mean total calories intake among the sample was $1410 \pm 370.1$, with $58.2 \pm 9.2 \%$ being carbohydrates, $21.9 \pm 4.7 \%$ proteins, and $20 \pm 7.6 \%$ fat. No significant differences were found in forearm strength (hand-grip test) in all groups after interventions $(p>.05)$.

\section{Inflammatory markers}

Baseline post-hoc comparisons between those who finished the trial different interventions showed that TAU had the lowest values for TNF- $\alpha$ and IL-10 $(p<0.05)$. Table 2 shows the inflammatory markers and cognitive changes after the 14 weeks intervention. No significant treatment*time interactions were observed for IL-1 $\beta$ and IL-1ra levels over time within the four groups $(p<0.05)$, despite a non-significant decrease in CET and TAU groups for IL- $1 \beta$ levels $(d=0.6$ and $d=0.65$, respectively). Meanwhile, a trend to increase in IL-1ra concentration was observed for the CET $(d=0.62, p=.06)$. Taken together, these small changes resulted in a moderate decrease using the IL$1 \beta / \mathrm{IL}-1$ ra ratio in both the CET and TAU groups (CI 95\%: .001 to .16, $p=.04$, and CI 95\%: .036 to 
$.289, p=.01$, respectively). Despite no significant treatment*time interactions observed for TNF- $\alpha$ levels, univariate analysis showed a moderate decrease in CET $(p=.02, d=0.99)$ and CG $(p=.01$, $d=0.68$ ), whereas no significant change was seen in the other groups. Repeated measures ANOVA showed a significant treatment*time interaction for changes in IL-10 levels, where a decrease in CG was observed ( $F=12.189$, CI 95\%: .094 to $.351, p=.001)$, with no significant variations occurring in CET, TAU and CET+TAU. Taken together, these changes lead to a significant treatment*time interaction in TNF- $\alpha /$ IL-10 ratio $(F=4.265, p=.01)$, where a moderate decrease occurred in the CET group (CI 95\%: 279 to $1.724, p=.04$ ), and an increase in this ratio was observed for CG (CI 95\%: 1.30 to $-.02, p=.01)$. Plasma IL-6 decreased only in CET $(p=0.03)$, together with a decrease in the IL-6/IL10 (CI 95\%: .124 to 1.23, $p=.02$ ). No significant changes were observed in IL-17 levels for all groups between baseline and after 14 weeks (Table 2).

\section{Cognition:}

Significant time $\mathrm{x}$ treatment interactions were observed for $\operatorname{MMSE}(F=7.49, p<.01)$, where a moderate increase occurred in the CET+TAU group (CI 95\%: -3.987 to $-.922, p=.04$ ), while a decrease in CG score was observed (CI 95\%: .616 to 3.550, $p=.03$ ). No significant changes were observed after CET and TAU interventions $(p>.05)$.

\section{Peripheral markers of brain damage}

S100 $\beta$ and NSE: None of the interactions reached significance for $\mathrm{S} 100 \beta$ (F(df: 1, 40)=1.241, $p=.308$ ). However, adjusted plots of these analyses and univariate tests indicated a trend towards a decline in $\operatorname{CET}(p=.06 ; d=0.68)$, and a non-significant elevation in this parameter for controls $(p=.44)$ (Figure 2).

Multivariate stepwise backward regression analyses including the interleukins percent changes and baseline co-morbidity showed that there was a trend for changes in IL-1 $\beta$ and CCI to be independently associated with changes in S100 $\beta$. All other variables (IL-1ra, IL-6, TNF- $\alpha$ and IL10), were removed in these stepwise backward analyses and did not contribute to the model (Table 3). Repeated measures ANOVA revealed that there was only a time effect for NSE changes 
$(F=4.182, p=0.04)$, but no significant treatment*time interactions $(p>.05)$. Univariate comparisons showed no significant changes in CET, CET+TAU and CG in comparison to baseline, however, a moderate increase in NSE was observed in the TAU group after the 14-week intervention $(p=.03$, $d=0.96)$.

\section{DISCUSSION}

In this study, we investigated the effects of fourteen weeks of CET and supplementation with taurine, alone and combined, on immune parameters and peripheral markers of BBB permeability in institutionalized elderly women. Results showed that a CET intervention maintained concentrations of peripheral markers for BBB integrity and improved cytokine balance. The same magnitude of effect was not observed for the other interventions (except for a large effect of taurine in decreasing the IL-1 $/$ IL-1ra ratio). The supplementation with taurine together with exercise did not improve the immune parameters in comparison to the interventions alone, but significantly increased cognition which was not seen in the other groups. Our observations provide support for the ability of exercise to maintain BBB integrity and decrease chronic inflammation in elderly women, which might be relevant for public health programs in neurodegenerative disease prevention. As a naturalistic study considering preferences of participants for interventions, the study has additional value.

Participants in this study were individuals with average age slightly higher than that in other studies with exercise programs for elderly people (Etnier et al. 2006), however, our study was composed only by institutionalized elderly women who were supposed to be more debilitated and engaged in less physical activity than the community-dwelling population. Despite non-significant, the small differences in CCI at baseline between the four groups might be relevant for the magnitude of the effect of the interventions tested. It is possible that the exercise in this study was too vigorous for individuals with more comorbidities, leading them to attenuate the effort during exercise activities while reporting the same perceived exertion. 
The S100 $\beta$ protein is suggested as a peripheral marker of BBB permeability (Marchi et al. 2003, Kanner et al. 2003), being useful and relatively non-invasive for older adults (Mecocci et al. 1995, Chaves et al. 2010). Despite also being expressed in other tissues than brain, $\mathrm{S} 100 \beta$ is present primarily in astrocytes, and extracranial sources of this protein do not affect resting serum levels (Pham et al. 2010). Over expression of this protein occurs in neurodegenerative conditions, such as Alzheimer's and Parkinson Disease, as well as in traumatic brain injury and schizophrenia, situations where BBB permeability is compromised (Steiner et al. 2006, Michetti et al. 2012). Given the increasing longevity associated with increased incidence of neurodegenerative disorders, an intervention that has the potential to improve the BBB integrity is promising, particularly among the elderly population where exercise overall has consistently shown beneficial effects on brain function (Hogervorst and Clifford 2012, Northey et al. 2017).

The mechanism by which long-term exercise can maintain the permeability of the BBB in humans is still subject of debate, and until today the few studies investigating this were done using animal models. Guo and co-workers (Guo et al. 2008) pointed out that exercise could improve the BBB structure by ameliorating the integrity of the basal lamina, in association with a decreased expression of matrix-metalloproteinase-9. Exercise was also found to protect the striatal BBB in an animal model of diabetes (de Senna et al. 2015). The results of our study corroborate other findings where exercise produced an immunomodulatory effect, by reducing the pro-inflammatory and increasing the anti-inflammatory cytokines (Souza et al. 2016). Production of TNF- $\alpha$ directly affects the BBB permeability (Larochelle et al. 2011). Evidence has shown that physical exercise inhibited the production of pro-inflammatory cytokines, upregulated the regulatory $\mathrm{T}$ cells, and suppressed IL- 6 and TNF- $\alpha$ production associated with the inhibition of adhesion molecules and reestablishment of tight junction protein expression, thereby restraining BBB permeability (Mota et al. 2012, Souza et al. 2016). Our exercise results clearly showed a decrease in the IL-1ß/IL-1ra, IL6/IL10 and TNF- $\alpha$ /IL-10 ratios. Thus, one can put forward the hypothesis that these effects could contribute to the decrease in $\mathrm{S} 100 \beta$ after the intervention. Other mechanisms by which exercise can 
ameliorate the BBB integrity could be due to the modulatory redox status of sensitive small GTPase, an enzyme that is activated by rho kinase (Wolff et al. 2014). Activation of rho GTPases is related to the decrease in the expression of proteins from tight junction (such as claudin-5), and increase in BBB permeability through monocyte migration (Persidsky et al. 2006). Evidence has demonstrated that exercising mice had less activation of rho kinase than sedentary animals, supporting the idea that exercise practice could maintain the BBB integrity through these mechanisms (Wolff et al. 2015).

The anti-inflammatory and antioxidant effect of taurine might explain a slight decrease in S100 $\beta$ after interventions in supplemented groups. Myeloperoxidase can derivate production of hypochlorous acid $\left(\mathrm{HOCl}^{-}\right)$as a oxidative species and has previously been related to $\mathrm{BBB}$ breakdown (Üllen et al. 2013). Supplementation with taurine can abolish the formation of $\mathrm{HOCl}^{-}$ (Marcinkiewicz and Kontny 2014). It is thus plausible that ingestion of taurine for 14 weeks suppressed the MPO-oxidative pathway and contributed to maintain BBB integrity, beyond its potential to increase anti-inflammatory cytokines. Considering this result, it seems clear that one of the most important mechanisms by which both exercise and taurine can act on BBB integrity is via an immunomodulatory and antioxidant pathway. Indeed, in response to the intervention protocols, we explored the relationship between changes in cytokines and S100 $\beta$ over time. Our results demonstrated that group membership determined changes between interventions and controls in this parameter, while there was also a trend for baseline CCI and change in IL-1 $\beta$ to be independently associated with change in S100ß. Despite not inferential, this result suggests the possibility that fluctuations in $\mathrm{BBB}$ integrity may be related to modulation of inflammatory cytokines, which is consistent to those investigations in animal model mentioned above (Mota et al. 2012, Souza et al. 2016).

Taurine, is essential to the normal growth and development of the nervous system, being related to neurogenesis, differentiation and neuroprotection (Gebara et al. 2015). The NSE in brain, is involved in the same processes, including maturation of neuronal cells (Isgrò et al. 2015). Since 
taurine can be transported across the BBB through B-amino acid transporter, it could induce neurotrophic factors by increasing the expression of NSE (Liu et al. 2013). It's plausible that an increased CNS formation of NSE induced by taurine could lead to an augmented efflux to peripheral circulation, increasing their serum levels as observed in TAU group. However, this mechanism need to be further exploited in humans.

Regarding differences in TNF- $\alpha$, IL-10 and TNF- $\alpha /$ IL-10 ratio, it seems that exercise is more likely to induce an anti-inflammatory environment rather than decrease inflammatory cytokine production. In fact, previous data with elderly individuals showed that twenty-eight weeks of exercise intervention was more effective in increasing IL-10 levels rather than decreasing TNF- $\alpha$ levels (Chupel et al. 2017). This effect may be mediated by an increase of production and release of anti-inflammatory cytokines by the repeated bouts of exercise from muscle (myokines), or reduced expression of Toll-like receptors in monocytes and macrophages (Gleeson et al. 2011). Meanwhile, groups who did not engage in exercise (TAU and CG) tended to show reduced IL-10 values over time. The mechanism by which exercise training decreased IL-6 concentration is generally attributed to a reduction in fat mass and/or via decrease formation of pro-inflammatory cytokines from peripheral mononuclear (PMN) cells. Since adipose tissue is a source of circulating IL-6, a reduction in fat mass could led to a decreased release of this cytokine into the blood (Gleeson et al. 2011). However, our intervention did not reduce the body mass in all groups, making unlikely that the reduction observed could be attributed to loss of fat mass alone. Since decreases in IL-1ß/IL1 ra, IL-6/IL-10 and TNF- $\alpha /$ IL-10 ratios were observed in CET, its plausible to think that reduction in IL-6 was influenced mainly by decreased cytokine production by PMN, which is consistent with other studies with elderly individuals (Kohut et al. 2006).

Results also showed that the combination of exercise and taurine promoted major responses in global cognition. When exercise or taurine supplementation were applied by themselves, maintenance of cognitive levels occurred and there were no significant changes. In contrast, cognitive function in $\mathrm{CG}$ declined after care as usual. The effects of exercise on global cognition 
have been previously reviewed, and generally implementation of several exercise programs have shown improvement in overall cognition (Hogervorst and Clifford 2012). However, different types of exercise and adherence, as well as the baseline cognitive level of the participants, may have introduced bias (Etnier et al. 2006). Effects of exercise were most strongly seen in elderly women (Hogervorst and Clifford 2012) which could explain the results of the present study which did not include men.

The mechanisms by which exercise improves cognition are many, and ranges from improvements in immunity (Stranahan et al. 2012), and/or inducing neurotrophic factors (Intlekofer and Cotman 2013). As mentioned, the groups where improved cognition occurred presented simultaneously with a better immune balance, which might explain why the effect on cognition was strongest in the combined treatment arm. A recent study has showed interaction between peripheral immunity and the CNS through the BBB, elucidating the fact that a decrease in blood pro-inflammatory cytokine could result in maintenance (or even, increase), of cognition (Engelhardt et al. 2017, Bradburn et al. 2018).

In animal models, taurine has been shown to protect or even improve cognition (Vohra and Hui 2000). Analogues of taurine such as homotaurine, have been proposed for therapeutic applications due its anti-amyloid activity (Caltagirone et al. 2012). This effect might be relevant for treatment of severe cognitive impairment, such as Alzheimer Disease. Due the similarity with GABA, taurine is known to act as an inhibitory neurotransmitter and neuromodulator (Ripps and Shen 2012). This involvement may explain the significance of taurine on cognitive function, since GABA influences cognition (Gabriella and Giovanna 2010). The importance of supplementation with taurine in the elderly could be explained due to the age-dependent reduction in levels. Even in healthy elderly there is a $49 \%$ decrease in plasma taurine concentration in comparison with middle-aged individuals (Jeevanandam et al. 1990), which may justify supplementation in elderly populations. Although physiological levels of taurine achieved by supplementation could generate several neuroendocrine 
responses in the healthy brain, further research is needed to investigate its effect in pathological conditions.

\section{CONCLUSION}

Despite some limitations (such as a small sample size), there are several important issues addressed by this naturalistic study. Firstly, our study suggests that exercise could help prevent the BBB leakage, even in advanced age. Secondly, this effect - at least in part, is probably mediated by immune changes, but this mechanism needs to be further investigated. In addition, taurine demonstrated small beneficial effects, but appears to be important as an auxiliary nutritional supplement to exercise to improve cognition in institutionalized elderly women.

\section{ACKNOWLEDGEMENTS}

The authors would like to thank the institution that accepted to participate in the study: Santa Casa de Misericórdia de Cantanhede, were this project was developed, and to all staff who volunteering help during all procedures. The authors would also like to thank the students Carla Cristóvão, Fábio Direito, Tais Rieping and Nelba Souza for help with exercise implementation and data collection.

\section{FOUNDING}

This work was partly supported by FEDER funding through COMPETE and the National funding through FCT - Portuguese Foundation for Science and Technology by grant [PTDC/DTPDES/0154/2012](UID/PTD/04213/2016); Chupel, Minuzzi and Furtado were financed by grant from CAPES/CNPQ (BEX: 13642-13/8, 1417/3-4 and 11929-13/8, respectively), Ministry of Education, Brazil.

\section{CONFLICT OF INTEREST}


The authors declare that the results of this study are presented clearly, honestly, and without fabrication, falsification, or inappropriate data manipulation, with no conflict of interest.

\section{REFERENCES}

Abbott, N.J. 2000. Inflammatory mediators and modulation of blood-brain barrier permeability. Cell. Mol. Neurobiol. 20(2): 131-47.

Ahmadian, M., Roshan, V.D., Aslani, E., and Stannard, S.R. 2017. Taurine supplementation has anti-atherogenic and anti-inflammatory effects before and after incremental exercise in heart failure. Ther. Adv. Cardiovasc. Dis. 11(7): 185-194. doi:10.1177/1753944717711138.

Beavers, K.M., Brinkley, T.E., and Nicklas, B.J. 2010. Effect of exercise training on chronic inflammation. Clin. Chim. Acta, 411(11-12): 785-793. doi:10.1016/j.cca.2010.02.069.

Bradburn, S., Sarginson, J., and Murgatroyd, C.A. 2018. Association of Peripheral Interleukin-6 with Global Cognitive Decline in Non-demented Adults: A Meta-Analysis of Prospective Studies. Front. Aging Neurosci. 9(January). doi:10.3389/fnagi.2017.00438.

Caltagirone, C., Ferrannini, L., Marchionni, N., Nappi, G., Scapagnini, G., and Trabucchi, M. 2012. The potential protective effect of tramiprosate (homotaurine) against Alzheimer's disease: a review. Aging Clin. Exp. Res. 24(6): 580-7. doi:10.3275/8585.

Charlson, M., Szatrowski, T.P., Peterson, J., and Gold, J. 1994. Validation of a combined comorbidity index. J. Clin. Epidemiol. 47(11): 1245-51.

Chaves, M.L., Camozzato, A.L., Ferreira, E.D., Piazenski, I., Kochhann, R., Dall’Igna, O., Mazzini, G.S., Souza, D.O., and Portela, L. V. 2010. Serum levels of S100B and NSE proteins in Alzheimer's disease patients. J. Neuroinflammation, 7: 6-12. doi:10.1186/1742-2094-7-6.

Cho, S.-H., Chen, J.A., Sayed, F., Ward, M.E., Gao, F., Nguyen, T.A., Krabbe, G., Sohn, P.D., Lo, I., Minami, S., Devidze, N., Zhou, Y., Coppola, G., and Gan, L. 2015. SIRT1 Deficiency in Microglia Contributes to Cognitive Decline in Aging and Neurodegeneration via Epigenetic Regulation of IL-1 . J. Neurosci. 35(2): 807-818. doi:10.1523/JNEUROSCI.2939-14.2015. 
Chupel, M.U., Direito, F., Furtado, G.E., Minuzzi, L.G., Filipa, M., Colado, J., Ferreira, J.P., Filaire, E., and Teixeira, A.M. 2017. Strength Training Decreases Inflammation and Increases Cognition and Physical Fitness in Older Women with Cognitive Impairment. Front. Physiol. 8(June): 1-13. doi:10.3389/fphys.2017.00377.

Dadas, A., Washington, J., Marchi, N., and Janigro, D. 2016. Improving the clinical management of traumatic brain injury through the pharmacokinetic modeling of peripheral blood biomarkers. Fluids Barriers CNS. 13(1): 21. BioMed Central. doi:10.1186/s12987-016-0045-y.

Elwood, E., Lim, Z., Naveed, H., and Galea, I. 2017. The effect of systemic inflammation on human brain barrier function. Brain Behav. Immun. 62: 35-40. doi:10.1016/j.bbi.2016.10.020.

Engelhardt, B., Vajkoczy, P., and Weller, R.O. 2017. The movers and shapers in immune privilege of the CNS. Nat. Immunol. 18(2): 123-131. doi:10.1038/ni.3666.

Etnier, J.L., Nowell, P.M., Landers, D.M., and Sibley, B.A. 2006. A meta-regression to examine the relationship between aerobic fitness and cognitive performance. Brain Res. Rev. 52(1): 119130. doi:10.1016/j.brainresrev.2006.01.002.

Folstein, M.F., Folstein, S.E., and McHugh, P.R. 1975. A practical state method for grading the cognitive state of patients for the clinician. J. Psychiatr. Res. 12(3): 189-198. doi:10.1016/0022-3956(75)90026-6.

Gabriella, G., and Giovanna, C. 2010. $\gamma$-Aminobutyric Acid Type A (GABAA) Receptor Subtype Inverse Agonists as Therapeutic Agents in Cognition. In Methods in Enzymology. Elsevier Inc. pp. 197-211.

Gebara, E., Udry, F., Sultan, S., and Toni, N. 2015. Taurine increases hippocampal neurogenesis in aging mice. Stem Cell Res. 14(3): 369-379. doi:10.1016/j.scr.2015.04.001.

Gleeson, M., Bishop, N.C., Stensel, D.J., Lindley, M.R., Mastana, S.S., and Nimmo, M. a. 2011. The anti-inflammatory effects of exercise: mechanisms and implications for the prevention and treatment of disease. Nat. Rev. Immunol. 11(9): 607-15. doi:10.1038/nri3041.

Guigoz, Y., Vellas, B., and Garry, P.J. 1996. Assessing the Nutritional Status of the Elderly: The 
Mini Nutritional Assessment as Part of the Geriatric Evaluation. Nutr. Rev. 54(1): S59-S65. doi:10.1111/j.1753-4887.1996.tb03793.x.

Guo, M., Cox, B., Mahale, S., Davis, W., Carranza, a, Hayes, K., Sprague, S., Jimenez, D., and Ding, Y. 2008. Pre-ischemic exercise reduces matrix metalloproteinase-9 expression and ameliorates blood-brain barrier dysfunction in stroke. Neuroscience, 151(2): 340-51. doi:10.1016/j.neuroscience.2007.10.006.

Haque, A., Ray, S.K., Cox, A., and Banik, N.L. 2016. Neuron specific enolase: a promising therapeutic target in acute spinal cord injury. Metab. Brain Dis. 31(3): 487-495. Metabolic Brain Disease. doi:10.1007/s11011-016-9801-6.

Hogervorst, E., and Clifford, A. 2012. Exercise to Prevent Cognitive Decline and Alzheimer's disease: For Whom, When, What, and (most importantly) How Much? J. Alzheimer's Dis. Park. 2(3): 2-4. doi:10.4172/2161-0460.1000e117.

Hopkins, W.G., Marshall, S.W., Batterham, A.M., and Hanin, J. 2009. Progressive statistics for studies in sports medicine and exercise science. Med. Sci. Sports Exerc. 41(1): 3-12. doi:10.1249/MSS.0b013e31818cb278.

Intlekofer, K.A., and Cotman, C.W. 2013. Exercise counteracts declining hippocampal function in aging and Alzheimer's disease. Neurobiol. Dis. 57: 47-55. Elsevier B.V. doi:10.1016/j.nbd.2012.06.011.

Isgrò, M.A., Bottoni, P., and Scatena, R. 2015. Neuron-Specific Enolase as a Biomarker: Biochemical and Clinical Aspects. In Advances in experimental medicine and biology. pp. 125-143. doi:10.1007/978-94-017-7215-0_9.

Jeevanandam, M., Young, D.H., Ramias, L., and Schiller, W.R. 1990. Effect of major trauma on plasma free amino acid concentrations in geriatric patients. Am. J. Clin. Nutr. 51(6): 1040-5. Kanner, A. a, Marchi, N., Fazio, V., Mayberg, M.R., Koltz, M.T., Siomin, V., Stevens, G.H.J., Masaryk, T., Aumayr, B., Ayumar, B., Vogelbaum, M. a, Barnett, G.H., and Janigro, D. 2003. Serum S100beta: a noninvasive marker of blood-brain barrier function and brain lesions. 
Cancer, 97(11): 2806-13. doi:10.1002/cncr.11409.

Kohut, M.L., McCann, D.A., Russell, D.W., Konopka, D.N., Cunnick, J.E., Franke, W.D., Castillo, M.C., Reighard, A.E., and Vanderah, E. 2006. Aerobic exercise, but not flexibility/resistance exercise, reduces serum IL-18, CRP, and IL-6 independent of $\beta$-blockers, BMI, and psychosocial factors in older adults. Brain. Behav. Immun. 20(3): 201-209. doi:10.1016/j.bbi.2005.12.002.

Larochelle, C., Alvarez, J.I., and Prat, A. 2011. How do immune cells overcome the blood-brain barrier in multiple sclerosis? FEBS Lett. 585(23): 3770-3780. doi:10.1016/j.febslet.2011.04.066.

Liu, J., Liu, Y., Wang, X.-F., Chen, H., and Yang, N. 2013. Antenatal taurine supplementation improves cerebral neurogenesis in fetal rats with intrauterine growth restriction through the PKA-CREB signal pathway. Nutr. Neurosci. 16(6): 282-287. doi:10.1179/1476830513Y.0000000057.

Marchi, N., Cavaglia, M., Fazio, V., Bhudia, S., Hallene, K., and Janigro, D. 2004. Peripheral markers of blood-brain barrier damage. Clin. Chim. Acta, 342(1-2): 1-12. doi:10.1016/j.ccen.2003.12.008.

Marchi, N., Rasmussen, P., Kapural, M., Fazio, V., Kight, K., Mayberg, M.R., Kanner, A., Ayumar, B., Albensi, B., Cavaglia, M., and Janigro, D. 2003. Peripheral markers of brain damage and blood-brain barrier dysfunction. Restor. Neurol. Neurosci. 21(3-4): 109-21.

Marcinkiewicz, J., and Kontny, E. 2014. Taurine and inflammatory diseases. Amino Acids 46(1): 7-20. doi:10.1007/s00726-012-1361-4.

Mecocci, P., Parnetti, L., Romano, G., Scarelli, A., Chionne, F., Cecchetti, R., Polidori, M.C., Palumbo, B., Cherubini, A., and Senin, U. 1995. Serum anti-GFAP and anti-S100 autoantibodies in brain aging, Alzheimer's disease and vascular dementia. J.Neuroimmunol. 57(0165-5728): 165-170.

Michetti, F., Corvino, V., Geloso, M.C., Lattanzi, W., Bernardini, C., Serpero, L., and Gazzolo, D. 
2012. The S100B protein in biological fluids: More than a lifelong biomarker of brain distress. J. Neurochem. 120(5): 644-659. doi:10.1111/j.1471-4159.2011.07612.x.

Morgado, J., Rocha, C.S., Maruta, C., Guerreiro, M., and Martins, I.P. 2009. Novos Valores Normativos do Mini-Mental State Examination. Sinapse, 9(2): 10-16. doi:10.1016/j.mpsur.2015.05.002.

Mota, B.C., Pereira, L., Souza, M.A., Silva, L.F.A., Magni, D.V., Ferreira, A.P.O., Oliveira, M.S., Furian, A.F., Mazzardo-Martins, L., Silva, M.D. Da, Santos, A.R.S., Ferreira, J., Fighera, M.R., and Royes, L.F.F. 2012. Exercise pre-conditioning reduces brain inflammation and protects against toxicity induced by traumatic brain injury: behavioral and neurochemical approach. Neurotox. Res. 21(2): 175-84. doi:10.1007/s12640-011-9257-8.

Nelson, M.E., Rejeski, W.J., Blair, S.N., Duncan, P.W., Judge, J.O., King, A.C., Macera, C.A., and Castaneda-Sceppa, C. 2007. Physical Activity and Public Health in Older Adults: recommendation from the American College of Sports Medicine and the American Heart Association. Med. Sci. Sport. Exerc. 39(8): 1435-1445. doi:10.1249/mss.0b013e3180616aa2.

Northey, J.M., Cherbuin, N., Pumpa, K.L., Smee, D.J., and Rattray, B. 2017. Exercise interventions for cognitive function in adults older than 50: a systematic review with meta-analysis. Br. J. Sports Med. (3): bjsports-2016-096587. doi:10.1136/bjsports-2016-096587.

Persidsky, Y., Heilman, D., Haorah, J., Zelivyanskaya, M., Persidsky, R., Weber, G.A., Shimokawa, H., Kaibuchi, K., and Ikezu, T. 2006. Rho-mediated regulation of tight junctions during monocyte migration across the blood-brain barrier in HIV-1 encephalitis (HIVE). Blood, 107(12): 4770-4780. doi:10.1182/blood-2005-11-4721.

Pham, N., Fazio, V., Cucullo, L., Teng, Q., Biberthaler, P., Bazarian, J.J., and Janigro, D. 2010. Extracranial sources of S100B do not affect serum levels. PLoS One, 5(9): e12691. doi:10.1371/journal.pone.0012691.

Redmond, H.P., Stapleton, P.P., Neary, P., and Bouchier-Hayes, D. 1998. Immunonutrition: the role of taurine. Nutrition, 14(7-8): 599-604. 
Ripps, H., and Shen, W. 2012. Review: taurine: a "very essential" amino acid. Mol. Vis. 18(November): 2673-86.

Să̆, S., Să̆, M.S., Tekeoğlu, I., Kamanl, A., Nas, K., and Acar, B.A. 2017. Central nervous system involvement in rheumatoid arthritis: possible role of chronic inflammation and tnf blocker therapy. Acta Neurol. Belg.: 1-7. doi:10.1007/s13760-017-0879-3.

de Senna, P.N., Xavier, L.L., Bagatini, P.B., Saur, L., Galland, F., Zanotto, C., Bernardi, C., Nardin, P., Goncalves, C.A., and Achaval, M. 2015. Physical training improves non-spatial memory, locomotor skills and the blood brain barrier in diabetic rats. Brain Res. 1618: 75-82. doi:10.1016/j.brainres.2015.05.026.

Shao, A., and Hathcock, J.N. 2008. Risk assessment for the amino acids taurine, 1-glutamine and 1arginine. Regul. Toxicol. Pharmacol. 50(3): 376-399. doi:10.1016/j.yrtph.2008.01.004.

Souza, P.S., Gonçalves, E.D., Pedroso, G.S., Farias, H.R., Junqueira, S.C., Marcon, R., Tuon, T., Cola, M., Silveira, P.C.L., Santos, A.R., Calixto, J.B., Souza, C.T., de Pinho, R.A., and Dutra, R.C. 2016. Physical Exercise Attenuates Experimental Autoimmune Encephalomyelitis by Inhibiting Peripheral Immune Response and Blood-Brain Barrier Disruption. Mol. Neurobiol.: 1-15. doi:10.1007/s12035-016-0014-0.

Steiner, J., Bielau, H., Bernstein, H.-G., Bogerts, B., and Wunderlich, M.T. 2006. Increased cerebrospinal fluid and serum levels of S100B in first-onset schizophrenia are not related to a degenerative release of glial fibrillar acidic protein, myelin basic protein and neurone-specific enolase from glia or neurones. J. Neurol. Neurosurg. Psychiatry, 77(11): 1284-1287. doi:10.1136/jnnp.2006.093427.

Stranahan, A.M., Martin, B., and Maudsley, S. 2012. Anti-inflammatory effects of physical activity in relationship to improved cognitive status in humans and mouse models of Alzheimer's disease. Curr. Alzheimer Res. 9(1): 86-92. doi:10.2174/156720512799015019.

Üllen, A., Singewald, E., Konya, V., Fauler, G., Reicher, H., Nusshold, C., Hammer, A., Kratky, D., Heinemann, A., Holzer, P., Malle, E., and Sattler, W. 2013. Myeloperoxidase-Derived 
Oxidants Induce Blood-Brain Barrier Dysfunction In Vitro and In Vivo. PLoS One, 8(5): e64034. doi:10.1371/journal.pone.0064034.

Vaughan, S., wallis, M., polit, D., steele, M., shum, D., and Morris, N. 2014. The effects of multimodal exercise on cognitive and physical functioning and brain-derived neurotrophic factor in older women: A randomised controlled trial. Age and Ageing, 43(5): 623-629. doi:10.1093/ageing/afu010.

Vohra, B.P., and Hui, X. 2000. Improvement of impaired memory in mice by taurine. Neural Plast. 7(4): 245-259. doi:10.1155/NP.2000.245.

Wolff, G., Balke, J.E., Andras, I.E., Park, M., and Toborek, M. 2014. Exercise Modulates RedoxSensitive Small GTPase Activity in the Brain Microvasculature in a Model of Brain Metastasis Formation. PLoS One, 9(5): 1-8. doi:10.1371/journal.pone.0097033.

Wolff, G., Davidson, S.J., Wrobel, J.K., and Toborek, M. 2015. Exercise maintains blood-brain barrier integrity during early stages of brain metastasis formation. Biochem. Biophys. Res. Commun. 463(4): 811-817. doi:10.1016/j.bbrc.2015.04.153. 


\section{TABLES}

Table 1. Participants characteristics at baseline

\begin{tabular}{c|c|c|c|c|c|c|c}
\hline Groups & Age (years) & Height $(\mathrm{cm})$ & Weight $(\mathrm{Kg})$ & BMI $\left(\mathrm{Kg} / \mathrm{m}^{2}\right)$ & Systolic BP $(\mathrm{mmHg})$ & $\begin{array}{c}\text { Diastolic BP } \\
(\mathrm{mmHg})\end{array}$ & $\begin{array}{c}\text { CCI } \\
\text { CET }(n=13)\end{array}$ \\
\hline TAU $(n=12)$ & $83.5(7.3)$ & $155.4(6.0)$ & $65.2(11.3)$ & $27.2(3.8)$ & $140.9(19.8)$ & $69.7(12.6)$ & $6.6(1.5)$ \\
\hline CET+TAU $(n=11)$ & $83.8(8.6)$ & $157.7(7.4)$ & $71(11.3)$ & $27.1(3.2)$ & $139.9(18.8)$ & $72.4(8.1)$ & $6.8(0.9)$ \\
\hline CG $(n=12)$ & $82(7.5)$ & $150.5(2.8)$ & $62.6(9.5)$ & $28.1(4.5)$ & $139.5(13.5)$ & $64.3(13.2)$ & $7(1.7)$ \\
\hline
\end{tabular}

Values are expressed in mean(SD). CET: combined exercise training; TAU: taurine supplementation; CET+TAU: combined exercise training + taurine supplementation; CG: control group; BMI: body mass index, BP: blood pressure, CCI: Charlson Comorbidity Index (score). 
Table 2. Cytokines and cognitive changes.

\begin{tabular}{|c|c|c|c|c|c|c|c|}
\hline Groups & Baseline & After 14-weeks & Effects & $F$ & Overall $p^{\text {a }}$ & $p^{\mathrm{b}}$ (0 to 14-weeks) & $\mathrm{ES}^{\mathrm{c}}$ \\
\hline \multicolumn{8}{|c|}{ IL-1 $\beta(\mathrm{pg} / \mathrm{mL})$} \\
\hline CET & $4.5(1.2)$ & $4.0(0.3)$ & & & & 0.142 & 0.60 \\
\hline TAU & $4.0(0.2)$ & $3.8(0.5)$ & Time & 2.905 & 0.096 & 0.128 & 0.65 \\
\hline $\mathrm{CET}+\mathrm{TAU}$ & $4.3(0.4)$ & $4.3(0.5)$ & Time* group & 0.663 & 0.580 & 0.656 & 0.19 \\
\hline $\mathrm{CG}$ & $4.6(0.9)$ & $4.2(0.6)$ & & & & 0.48 & 0.29 \\
\hline \multicolumn{8}{|c|}{ IL-1ra (pg/mL) } \\
\hline CET & $26.3(13.6)$ & $35.1(13.7)$ & & & & 0.067 & 0.62 \\
\hline TAU & $29.1(19.3)$ & $28.0(10.0)$ & Time & 3.443 & 0.070 & 0.742 & 0.36 \\
\hline $\mathrm{CET}+\mathrm{TAU}$ & $25.6(16.0)$ & $24.9(8.1)$ & Time*group & 1.113 & 0.354 & 0.51 & 0.04 \\
\hline $\mathrm{CG}$ & $26.8(12.9)$ & $28.4(10.2)$ & & & & 0.719 & 0.26 \\
\hline \multicolumn{8}{|c|}{ IL-1 $\beta /$ IL-1 ra ratio } \\
\hline CET & $0.2(0.1)$ & $0.1(0.0)$ & & & & 0.047 & 0.88 \\
\hline TAU & $0.3(0.1)$ & $0.1(0.0)$ & Time & 10.487 & 0.002 & 0.016 & 1.00 \\
\hline $\mathrm{CET}+\mathrm{TAU}$ & $0.2(0.1)$ & $0.1(0.0)$ & Time*group & 2.560 & 0.067 & 0.286 & 0.23 \\
\hline $\mathrm{CG}$ & $0.2(0.1)$ & $0.2(0.2)$ & & & & 0.995 & 0.26 \\
\hline \multicolumn{8}{|c|}{ TNF- $\alpha(\mathrm{pg} / \mathrm{mL})$} \\
\hline CET & $12.8(7.5)$ & $9.7(3.9)$ & & & & 0.038 & 0.99 \\
\hline TAU & $8.6(6.4)$ & $7.7(5.2)$ & Time & 2.067 & 0.158 & 0.452 & 0.57 \\
\hline CET+TAU & $16.8(8.1)$ & $18.3(6.9)$ & Time*group & 2.238 & 0.097 & 0.383 & 0.27 \\
\hline $\mathrm{CG}$ & $12.8(5.6)$ & $9.6(3.7)$ & & & & 0.094 & 0.68 \\
\hline \multicolumn{8}{|c|}{ IL-10 (pg/mL) } \\
\hline CET & $3.0(1.9)$ & $3.3(1.9)$ & & & & 0.641 & 0.07 \\
\hline TAU & $2.2(1.1)^{\#}$ & $2.0(1.1)$ & Time & 2.837 & 0.099 & 0.289 & 0.32 \\
\hline $\mathrm{CET}+\mathrm{TAU}$ & $3.5(1.1)$ & $3.5(0.7)$ & Time* group & 3.470 & 0.024 & 0.711 & 0.11 \\
\hline $\mathrm{CG}$ & $4.4(1.6)$ & $2.2(1.5)$ & & & & 0.01 & 1.30 \\
\hline \multicolumn{8}{|c|}{ TNF/IL-10 ratio } \\
\hline CET & $4.6(1.6)^{\#}$ & $3.6(1.7)$ & & & & 0.039 & 0.85 \\
\hline TAU & $3.6(1.3)$ & $4.0(1.6)$ & Time & 0.307 & 0.583 & 0.374 & 0.43 \\
\hline $\mathrm{CET}+\mathrm{TAU}$ & $4.6(1.7)^{\#}$ & $5.1(1.3)$ & Time*group & 4.265 & 0.010 & 0.310 & 0.51 \\
\hline $\mathrm{CG}$ & $3.1(1.5)$ & $3.7(1.5)$ & & & & 0.042 & 1.14 \\
\hline \multicolumn{8}{|l|}{ IL-6 (pg/mL) } \\
\hline CET & $7.0(5.4)$ & $4.9(5.7)$ & & & & 0.036 & 0.76 \\
\hline TAU & $4.4(2.2)$ & $4.4(2.5)$ & Time & 2.669 & 0.109 & 0.619 & 0.04 \\
\hline CET+TAU & $8.6(10.2)$ & $8.6(7.7)$ & Time*group & 2.521 & 0.07 & 0.227 & 0.27 \\
\hline
\end{tabular}




\begin{tabular}{|c|c|c|c|c|c|c|c|}
\hline $\mathrm{CG}$ & 7.7(9.4) & $4.4(2.0)$ & & & & 0.298 & 0.50 \\
\hline \multicolumn{8}{|c|}{ IL-6/IL-10 ratio } \\
\hline CET & $2.0(1.1)$ & $1.3(1.0)$ & & & & 0.02 & 1.11 \\
\hline TAU & $2.5(1.8)$ & $2.8(2.3)$ & Time & 0.003 & 0.954 & 0.340 & 0.43 \\
\hline $\mathrm{CET}+\mathrm{TAU}$ & $2.5(3.4)$ & $2.4(2.1)$ & Time* group & 1.414 & 0.251 & 0.841 & 0.64 \\
\hline $\mathrm{CG}$ & $1.7(1.6)$ & $2.3(2.9)$ & & & & 0.478 & 0.43 \\
\hline \multicolumn{8}{|c|}{ IL-17 (pg/mL) } \\
\hline CET & $10.3(6.4)^{\#}$ & 9.1(4.6) & & & & 0.507 & 0.26 \\
\hline TAU & $17.8(9.6)$ & $14.9(6.5)$ & Time & 0.705 & 0.406 & 0.238 & 0.50 \\
\hline $\mathrm{CET}+\mathrm{TAU}$ & $17.4(10.7)$ & $16.3(12.2)$ & Time*group & 0.269 & 0.848 & 0.789 & 0.11 \\
\hline CG & $18.4(12.4)$ & $19.0(13.5)$ & & & & 0.638 & 0.19 \\
\hline \multicolumn{8}{|c|}{ MMSE (score) } \\
\hline CET & $20.2(5.8)$ & $21.7(5.0)$ & & & & 0.109 & 0.64 \\
\hline TAU & $18.6(5.8)$ & $18.2(5.8)$ & Time & 0.943 & 0.337 & 0.318 & 0.43 \\
\hline $\mathrm{CET}+\mathrm{TAU}$ & $21.3(4.5)$ & $23.8(3.6)$ & Time*group & 7.490 & 0.000 & 0.016 & 1.06 \\
\hline CG & $19.4(6.3)$ & $17.3(4.9)$ & & & & 0.015 & 0.98 \\
\hline
\end{tabular}

Values are presented in Mean(SD); ${ }^{a}$ Two-sided significance of differences across time and between the groups (repeated measures ANOVA); ${ }^{b}$ Two-sided significance of within differences between baseline and 14-week assessments (paired t-test); ${ }^{\mathrm{c}}$ Within effect size represented by Cohen's $d$ for changes in means; ${ }^{\#}$ Different from control group at baseline; Values of significance $(p<0.05)$ are highlighted in bold. 
Table 3. Multiple regression analysis for changes in S100ß

\begin{tabular}{lcccc}
\cline { 2 - 5 } & \multicolumn{4}{c}{$\Delta \mathrm{S} 100 \beta$} \\
\cline { 2 - 5 }$\Delta \mathrm{R}{ }^{2}$ & $\beta$ coefficient & $p$ value & $p$ ANOVA \\
\hline IIL-17 & & .278 & .056 & \\
CCI baseline & 0.163 & .205 & .162 & .048 \\
\hline
\end{tabular}

Note: unadjusted backward multiple regression analysis; changes in other cytokines (IL-6, IL-10, TNF- $\alpha$, and IL-1ra) did not contribute to the model and were removed in this analysis. 


\section{FIGURE CAPTIONS}

Figure 1. CONSORT (Consolidated Standards of Reporting Trials) of the study design. CET: combined exercise training; TAU: taurine supplementation; CET+TAU: combined exercise training plus taurine supplementation; CG: control group

Figure 2. A) S100ß; B) Neuronal-specific enolase (NSE); Lines represented means with standard error bars. With black lines: $\mathrm{CET}=$ combined exercise training; $\mathrm{TAU}=$ taurine supplementation; $\mathrm{CET}+\mathrm{TAU}=$ combined exercise training plus taurine supplementation; With grey line: $\mathrm{CG}=$ control group; T0: baseline; T1: after 14 weeks; $* p<0.05$ 


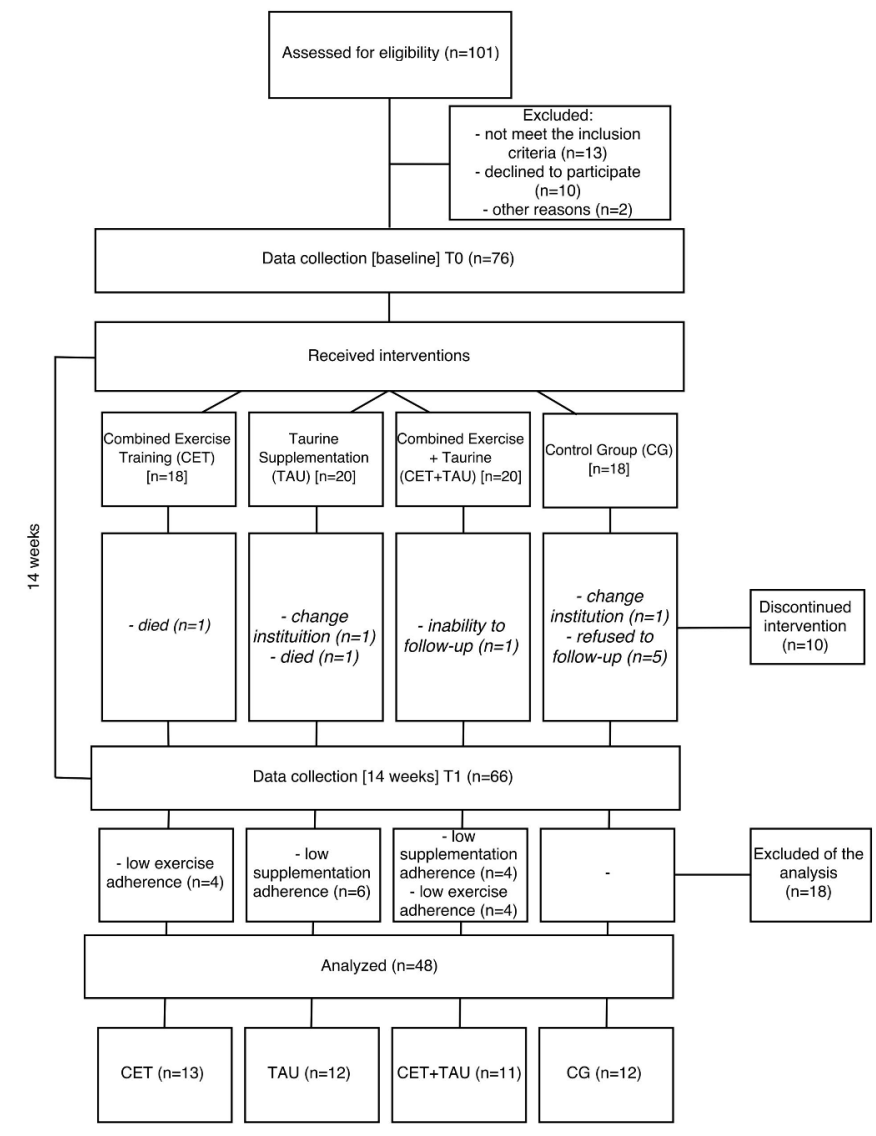

CONSORT (Consolidated Standards of Reporting Trials) of the study design. CET: combined exercise training; TAU: taurine supplementation; CET+TAU: combined exercise training plus taurine supplementation; CG: control group 

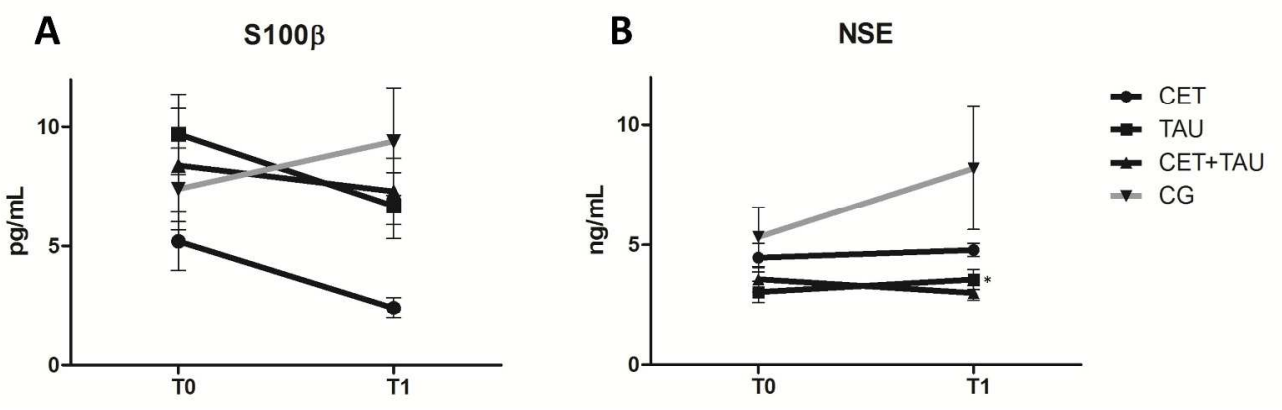

\begin{abstract}
A) S100ß; B) Neuronal-specific enolase (NSE); Lines represented means with standard error bars. With black lines: $\mathrm{CET}=$ combined exercise training; $\mathrm{TAU}=$ taurine supplementation; $\mathrm{CET}+\mathrm{TAU}=$ combined exercise training plus taurine supplementation; With grey line: $C G=$ control group; T0: baseline; T1: after 14 weeks; * $\mathrm{p}<0.05$
\end{abstract}

$338 \times 109 \mathrm{~mm}(300 \times 300$ DPI $)$ 